\title{
Lysis of Protoplasts of Micrococcus lysodeikticus by Alcohols
}

\author{
By A. R. GILBY* and the late A. V. FEW \\ Department of Colloid Science, University of Cambridge
}

(Received 7 December 1959)

\begin{abstract}
SUMMARY
The time course of lysis of protoplasts of Micrococcus lysodeikticus by $n$-ethanol, $n$-propanol, $n$-butanol and isoamyl alcohol was studied spectrophotometrically. The relative activity for lysis increased with the molecular weight of the alcohol used. Equal degrees of lysis resulted at equal thermodynamic activities of the alcohols. The high lytic concentrations of ethanol and propanol produced side effects which obscured the lysis/time curves. $n$-Butanol showed time delays before the onset of lysis; the significance of this is discussed in terms of protoplast-membrane structure. Because of similarities with lysis by cationic detergents and with the extraction of serum lipids in the presence of alcohols, it is concluded that the alcohols tested acted on the lipid component of the protoplast membrane and that lysis resulted from the disruption of membrane permeability.
\end{abstract}

\section{INTRODUCTION}

As bactericidal agents alcohols are much less effective than ionic detergents; the optimum bactericidal concentration of ethanol is usually between 30 and $50 \%$. The germicidal action of the shorter-chain alcohols increases with molecular weight. These agents are believed to act by dissolving in the lipid layer of the cell membrane and breaking down its structure by solvent action (Danielli, 1951). Mitchell (1953) showed that, above a critical concentration, butanol caused release of intracellular phosphate from certain bacteria. Most of the work on the lytic action of alcohols has been on erythrocytes. From comparative studies of haemolysis and monolayer penetration, Pethica \& Schulman (1953) concluded that alcohols haemolyse by a non-specific detergent process dependent upon a critical lowering of interfacial tension. While this applies at fairly rapid rates of haemolysis, the effect in general is more complicated since even very low concentrations of alcohols will eventually cause haemolysis (J. F. Hoffman, private communication). The adsorption of the alcohol is but the first step in a chain of processes. Pethica (1958) reviewed some possible mechanisms for the action of butanol on some membranes. Previous work (e.g. Salton, 1957; Gilby \& Few, 1960) dealt with aspects of lysis of bacterial protoplasts by ionic detergents. As examples of non-ionic soluble compounds, we have now studied short chain alcohols. Protoplasts of Micrococcus lysodeikticus were studied during lysis by a series of primary $n$-alkyl chain alcohols and by one secondary alcohol. The technique used allowed the reagent to react directly with the protoplast membrane free from an intervening cell wall.

* Present address : Division of Entomology, C.S.I.R.O., Canberra, A. C. T., Australia. 


\section{METHODS}

Compounds used. The $n$ primary alcohols used were ethanol, propanol, butanol and pentanol. These compounds (initially of A.R. quality) were purified by fractional distillation and the major fractions were collected over a range of less than $0 \cdot 1^{\circ}$. Preliminary experiments indicated that rigorous purity was essential as a small amount of higher molecular weight material could dominate the results. Some experiments were also performed with a fractionated sample of isoamyl alcohol.

Organisms used and protoplast formation. Micrococcus lysodeikticus (NCTC 2665) was grown on a beef tryptic-digest agar medium. The conditions in the controlled treatment with lysozyme to yield protoplasts and protoplast membranes were those of the preceding paper (Gilby \& Few, 1960). Protoplasts were stabilized in M-sucrose $+0.05 \mathrm{M}-\mathrm{NaCl}$ solution.

Spectrophotometric determination of lysis. The methods used were similar to those used with ionic detergents (Gilby \& Few, 1960). The optical density at wavelength $500 \mathrm{~m} \mu$ was measured during the reaction of protoplast suspension with alcohol at $22 \pm 1^{\circ}$. The concentration of protoplasts corresponded to $0.5 \mathrm{mg}$. dry wt. $/ \mathrm{ml}$., and the reaction medium (at $\mathrm{pH} 6 \cdot 3 \pm 0 \cdot 1$ ) contained $\mathrm{M}$-sucrose $+0.05 \mathrm{M}-\mathrm{NaCl}$. The usual precautions were taken to avoid spurious damage to protoplasts. In the range of alcohol and protoplast concentrations used, the rate of lysis was independent of cell concentration.

\section{RESULTS}

The activity of alcohols in protoplast lysis

The optical density/time curves for lysis are given in Figs. 1-3. The concentrations of ethanol in Fig. 1 required to produce lysis are several times molar. These high concentrations of ethanol caused precipitation of intracellular constituents of protoplasts and, as a result, the final optical densities $(c .0 \cdot 1)$ obtained on complete lysis by ethanol were larger than those obtained for lysis by higher alcohols. All concentrations of ethanol used gave rise to large increases in optical density in the initial stages of lysis. The lysis of protoplasts was more rapid at higher ethanol concentrations. With propanol (Fig. 2) the curves were broadly similar to those obtained with ethanol; the initial increases in optical density were smaller than the increases produced by ethanol. On complete lysis, the limiting value of optical density was near that corresponding to complete lysis by other means (osmotic explosion or ionic detergents). Thus the complication of precipitation with ethanol was not present in this range of propanol concentrations. In the results for butanol given in Fig. 3 the initial increases in optical density were very small. These curves suggested that there was a delay period before the onset of lysis and that the duration of the delay was decreased by increasing concentrations of butanol; there were no precipitation effects. With $n$-amyl alcohol it was impracticable to follow lysis since only extremely slow changes in optical density occurred, even at complete saturation. Isoamyl alcohol gave lysis/time curves similar in nature to those for butanol (Fig. 3).

The relative activities of the alcohols were compared in a manner similar to that used for ionic detergents (Gilby \& Few, 1960). The decrease in optical density after $30 \mathrm{~min}$. treatment has been plotted in Fig. 4 as a function of detergent concentration. 


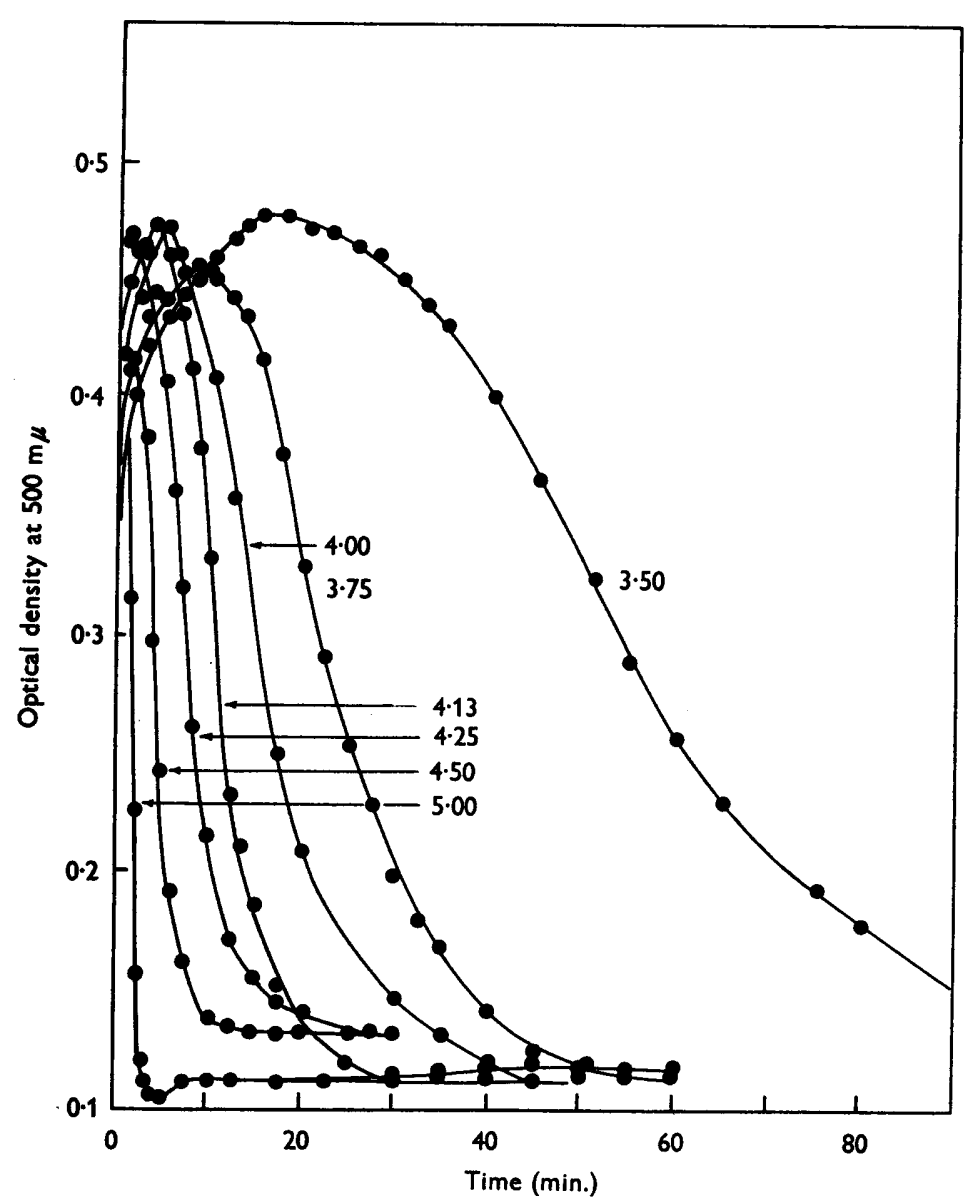

Fig. 1. Lysis of protoplasts of Micrococcus lysodeikticus by ethanol in M-sucrose+ $0.05 \mathrm{M}-\mathrm{NaCl}$ at concentrations of ethanol (M) given on curves.

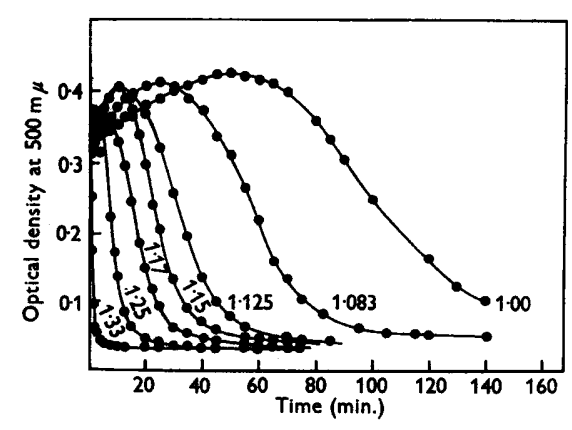

Fig. 2

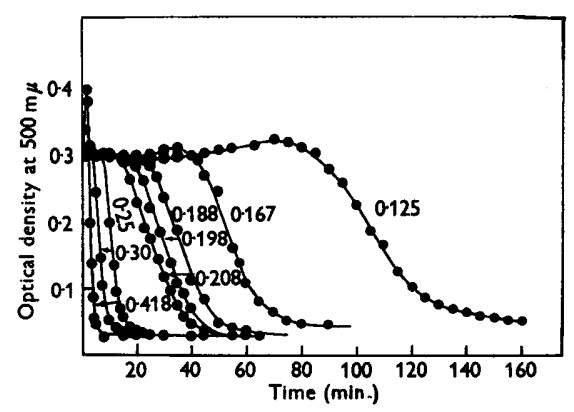

Fig. 3

Fig. 2. Lysis of protoplasts by $n$-propanol in $\mathrm{M}$-sucrose $+0.05 \mathrm{M}-\mathrm{NaCl}$ at concentrations of $n$-propanol (M) given on curves.

Fig. 3. Lysis of protoplasts by $n$-butanol in $\mathrm{M}$-sucrose $+0.05 \mathrm{M}-\mathrm{NaCl}$ at concentrations of $n$-butanol (M) given on curves. 
As previously, the protoplast lytic concentration was defined as that concentration of alcohol which in $30 \mathrm{~min}$. decreased the optical density of protoplast suspension by $90 \%$ of the loss observed on complete lysis. The curves in Fig. 4 for butanol and propanol are very steep and the determination of the protoplast lytic concentration was straightforward. With ethanol, the slope was greater, partly because of precipitation and the influences of the large initial increases in optical density. Extrapolation to the $90 \%$ line for other alcohols was not used. The definition was applied literally and the experimentally found specific $90 \%$ line was used to derive the protoplast lytic concentration as indicated.

The protoplast lytic concentrations of the alcohols and their activities relative to ethanol are given in Table 1. The lytic activity increased greatly with increase in chain length. A further comparison of the data was made by calculating the thermodynamic activity of each alcohol in the aqueous phase; the thermodynamic activity is a general expression of the 'effective' concentration of the alcohol. This differs from the actual concentration because of interactions between alcohol and solvent. The thermodynamic activities in Table 1 are only approximate because the values of the thermodynamic activity-coefficient used apply to solution in water, whereas the alcohols used here were dissolved in $\mathrm{M}$-sucrose $+0.05 \mathrm{M}-\mathrm{NaCl}$ solution. In the range of concentrations used, concentrations were substituted for mol fractions without serious inaccuracy in calculating thermodynamic activity. The values of thermodynamic activity calculated in this manner for the four alcohols are approximately equal. Considering the assumptions involved, the agreement is good. Thus, for the lysis of protoplasts by the aliphatic alcohols used, equal degrees of lysis resulted at equal thermodynamic activities of alcohol.

Table 1. Protoplast-lytic concentrations and thermodynamic data for alcohols

$\begin{array}{lcccc}\text { Protoplast } & \begin{array}{c}\text { Activity } \\ \text { lytic concn. } \\ \text { relative }\end{array} & \begin{array}{c}\text { Thermo- } \\ \text { to ethanol }\end{array} & W^{*} & \begin{array}{c}\text { Tynamic } \\ \text { dyctivity } \\ \left(A_{W}\right)\end{array} \\ \text { Ethanol } & 4.02 & 1 \cdot 0 & 3 \cdot 69 & 14 \cdot 8 \\ \text { 1-Propanol } & 1 \cdot 18 & 3 \cdot 4 & 11 \cdot 6 & 13 \cdot 5 \\ \text { 1-Butanol } & 0 \cdot 24 & 16 \cdot 8 & 51 \cdot 0 & 12 \cdot 2 \\ \text { 2-Pentanol } & 0.08 & 50.0 & 101 & 15 \cdot 3\end{array}$

* $W$ is thermodynamic activity coefficient in water of the alcohol at the protoplast-lytic concentration (from Butler, Thomson \& Maclennan, 1933, and from Butler, 1937).

The kinetics of lysis of protoplasts of Micrococcus lysodeikticus

The two general types of data used in the study of haemolysis are: (i) \% haemolysis/time curves; (ii) time/dilution curves (Ponder, 1948). The lysis/time curves in Figs. 1-3 for protoplast lysis are of the former type. Because of the complications with ethanol and propanol, attention will be confined here to lysis with butanol. The curves in Fig. 3 appear to fall into two groups. The curves at butanol concentrations of $0.25 \mathrm{M}$ and higher form a similar group whilst those at more dilute concentrations exhibit, after the initial delay period, a comparable course of reaction. By multiplying the time scale of the $0.208 \mathrm{M}$ curve by an appropriate factor, the curves for smaller dilutions can be accurately reproduced. A similar division of the kind of lysis into two concentration ranges is 
achieved by plotting delay times against butanol concentrations (Fig. 5). Here again the shape of the curves suggests that a change in character may occur at a butanol concentration of $c .0 .2 \mathrm{M}$. Other empirical plots of functions derived from the data reinforce this conclusion. As an example of the second type of treatment of data, a typical time/concentration curve which corresponds to $c .80 \%$ lysis by $n$-butanol is given in Fig. 5. Whilst kinetic equations can be derived to describe such curves, these equations contain constants of which the physical nature is obscure. Such formal equations would contribute little to an understanding of the principles involved.

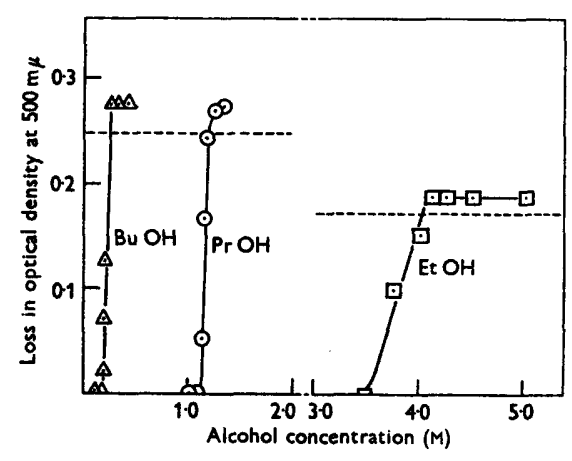

Fig. 4

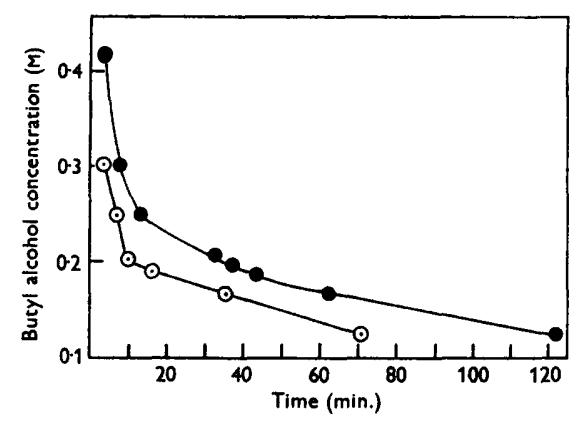

Fig. 5

Fig. 4. Decrease in optical density of suspensions of protoplasts of $M$. lysodeikticus after $\mathbf{3 0} \mathrm{min}$. lysis plotted as a function of alcohol concentration. Broken lines indicate $90 \%$ lysis.

Fig. 5. Time/concentration curves for lysis of protoplasts of $M$. lysodeikticus by $\boldsymbol{n}$-butanol in $\mathrm{M}$-sucrose $+0.05 \mathrm{M}-\mathrm{NaCl}$ solution. $=$ Time to reach optical density $0 \cdot 1, \odot=$ delay times before onset of lysis.

\section{DISCUSSION}

The lysis of protoplasts of Micrococcus lysodeikticus by alcohols bears some resemblance to the phenomenon of narcosis. At equilibrium the activity of alcohol must be the same in all phases and the activity in the external medium may be used to indicate the activity of alcohol in the protoplast membrane. If the activity coefficients of the alcohols in the sensitive site in the protoplast membrane are approximately the same (as they are in benzene; Butler \& Harrower, 1987), then the data in Table 1 indicate that equal concentrations of alcohol in the membrane will produce equal degrees of lysis. Brink \& Posternak (1948) suggested an empirical rule for narcosis which was analogous to this. Mullins (1954) argued that a better generalization would be that there were equal degrees of narcosis with equal volume fractions of narcotic in the membrane. In the present instance, when the thermodynamic activities at the protoplast lytic concentrations are multiplied by the molar volumes of the alcohols, the constancy of the products is slightly worse than that of the activities. However, there is no a priori reason why lysis and narcosis should have identical mechanisms. Indeed, narcosis has been suggested to result either from the prevention of the passage through the membrane of particular molecules or ions, depending on the degrees of occlusion by the narcotic, or else by action on intracellular lipids. Protoplast lysis, on the other hand, follows a breakdown in the 
impermeability of the protoplast membrane to solutes which normally protect the cell from osmotic rupture. Other results which present features similar to those of protoplast lysis have been encountered in studies on the extraction of lipids from serum by di-ethyl ether in the presence of alcohols. Machebouf \& Sandor (1932) obtained curves for the extraction of lipid which are similar to the protoplast lysis/ time curves, both in shape and in the occurrence of a delay period. The essential feature of their explanation was that di-ethyl ether cannot penetrate and dissolve the lipid until the alcohol has brought about far-reaching changes in organization of the lipid. Although Delaye (1935) found that butanol was not markedly more effective than ethanol, this could be due to partition between the ether solvent and the aqueous phase masking the greater activity of the butanol.

The alcohols interact with the protoplast membrane in different ways depending on the concentrations involved. The increases in optical density noted in the early stages of lysis with ethanol and propanol are greater than any observed to be produced by osmotic changes in volume (Gilby \& Few, 1959). These observations are more likely to result from changes in refractive index at the protoplast/medium interface by denaturation or dehydration of the outer protein carbohydrate layer of the protoplast shown to be present by electrophoresis (Few, Gilby \& Seaman, 1960). The effect is much less marked with butanol which has lower lytic concentrations. Uranyl nitrate caused inhibition of lysis over a similar range of concentrations to that which inhibited lysis by ionic detergents (Gilby \& Few, 1960). Detailed results for this effect have not been presented because replication was poor. Furthermore, protoplast membrane suspensions treated with $n$-butanol at concentrations over the range which produced lysis showed no change during $12 \mathrm{hr}$. In this way the action of the alcohol resembles that of cationic detergents and indicates that the protein matrix of the membrane is not broken down. From these results and the strong similarities to the serum-lipid extraction discussed above, it is concluded that the alcohols lyse protoplasts of Micrococcus lysodeikticus by their action on the lipid component of the protoplast membrane. Although the specific type of action on the lipid is uncertain, $n$-butanol may function by virtue of its property as a breaker of hydrogen bonds (Pethica, 1958). The shape of the butanol lysis/time curves can then be explained as follows. The delay period represents the time taken by the alcohol at a given concentration to penetrate and disrupt the organization of the protoplast-membrane lipid. Lysis then results from the changed permeability of the membrane and consequent loss of protection against osmotic explosion. The sigmoid shape of the curve, once lysis has begun, reflects the distribution of resistance to lysis of the protoplasts.

Attempts have been made to account quantitatively for the experimentally found delay periods with $n$-butanol, using models based on the above ideas. One such model in accord with data on the structure of the protoplast membrane is that the alcohol must diffuse through: (i) an initial barrier at the protein/aqueous interface in the protoplast surface; (ii) a relatively thick 'sandwich' layer of proteincarbohydrate; (iii) a second barrier at the protein/lipid interface. The equations based on this and other models to express the time taken for an alcohol to diffuse through different layers and to reach a critical concentration all suffer from the same fault in that they give delay times which do not increase fast enough as the concentration is decreased. Should the diffusion properties in the outer layers be 
time-dependent, this would complicate the model considerably. A successful quantitative account of the course of protoplast lysis must await a more detailed understanding of the events which precede and accompany the lysis. Evidence from other cells suggests that the penetration of the agent which causes lesion in the membrane is fast relative to the subsequent changes which result in lysis (e.g. volume changes in red cells; Parpart \& Green, 1951). Should this be so with protoplasts, it might explain the failure of the deductions from simple diffusion models. It is suggested that further studies on protoplasts in the presence of butanol during the pre-lytic delay period, e.g. investigations on the leakage of intracellular constituents and interrupted lysis, may be instructive.

We thank Dr J. H. Schulman for his interest in this work and the Commonwealth Scientific and Industrial Research Organization (Australia) for a Postgraduate Overseas Studentship to one of us (A. R. G.). The late A. V. Few was a member of the scientific staff of the Medical Research Council.

\section{REFERENCES}

Brink, F. \& Posternak, J. M. (1948). Thermodynamic analysis of the relative effectiveness of narcotics. J. cell. comp. Physiol. 32, 211.

Butler, J. A. V. (1937). The energy and entropy of hydration of organic compounds. Trans. Faraday Soc. 33, 229.

Butler, J. A. V. \& Harrower, P. (1937). The activity of some aliphatic alcohols and halides in non-polar solvents. Trans. Faraday Soc. 33, 171.

Butler, J. A. V., Thomson, D. W. \& Maclennan, W. H. (1933). The free energy of normal aliphatic alcohols in aqueous solution. J. chem. Soc. p. 674.

Danielli, J. F. (1951). The cell surface and cell physiology. In Cytology and Cell Physiology. Ed., G. H. Bourne, 2nd ed., p. 150. Oxford: Oxford University Press.

DELAYE, M. B. (1935). Contribution to the study of the stability of the lipoprotein complex in blood serum. Bull. Soc. Chim. Biol., Paris, 17, 927.

Few, A. V., Gilby, A. R. \& Seaman, G. V. F. (1960). An electrophoretic study of structural components of Micrococcus lysodeikticus. Biochim. biophys. Acta, 38, 130.

GrLby, A. R. \& FEw, A. V. (1959). Osmotic properties of protoplasts of Micrococcus lysodeikticus. J. gen. Microbiol. 20, 321.

GiLBY, A. R. \& FEw, A. V. (1960). Lysis of protoplasts of Micrococcus lysodeikticus by ionic detergents. J. gen. Microbiol. 23, 19.

MAchebouf, M. A. \& SANDor, G. (1932). Researches on the nature of the stability of the protein-lipid association. A study of the extraction of lipids by ether in the presence of alcohols. Bull. Soc. Chim. Biol., Paris, 14, 1168.

Mrtchell, P. (1953). Transport of phosphate across the surface of Micrococcus pyogenes: Nature of the cell 'inorganic phosphate'. J. gen. Microbiol. 9, 273.

Muluins, L. J. (1954). Some physical mechanisms in narcosis. Chem. Rev. 54, 289.

Parpart, A. K. \& Green, J. W. (1951). Potassium and sodium exchanges in rabbit red cells treated with $n$-butyl alcohol. J. cell. comp. Physiol. 38, 347.

Pethica, B. A. (1958). Lysis by physical and chemical methods. J. gen. Microbiol. 18, 473.

Pethica, B. A. \& Schulman, J. H. (1953). The physical chemistry of haemolysis by surfaceactive agents. Biochem. $J .53,17 \%$.

Ponder, E. (1948). Haemolysis and Related Phenomena. London: J. and A. Churchill Ltd.

SALTon, M. R. J. (1957). The action of lytic agents on the surface structures of the bacterial cell. Proc. 2nd. int. Congr. Surface Activity, 4, 245. 